\title{
Obstetrics and gynaecology in the era of COVID-19
}

\author{
Angela Mary George ${ }^{1 *}$, Daniel Manoj ${ }^{2}$, Sowmya Ramani $^{4}$, Lalropuii ${ }^{5}$, Murugan Timiri Palani ${ }^{3}$, \\ Ranjit Immanuel James ${ }^{2}$, Latif Rajesh Johnson ${ }^{2}$
}

\author{
${ }^{1}$ Department of Gynecologic Oncology, ${ }^{2}$ Department of Forensic Medicine and Toxicology, ${ }^{3}$ Department of Child \\ Health, Christian Medical College, Vellore, Tamil Nadu, India \\ ${ }^{4}$ Foetal Medicine Unit, Department of Obstetrics and Gynecology, Sri. Ramachandra Institute of Higher Education and \\ Research, Chennai, Tamil Nadu, India \\ ${ }^{5}$ National Health Mission, District Hospital, Mamit, Mizoram, India
}

Received: 29 August 2020

Accepted: 06 October 2020

\author{
*Correspondence: \\ Dr. Lalropuii, \\ E-mail:puitei06@gmail.com
}

Copyright: (C) the author(s), publisher and licensee Medip Academy. This is an open-access article distributed under the terms of the Creative Commons Attribution Non-Commercial License, which permits unrestricted non-commercial use, distribution, and reproduction in any medium, provided the original work is properly cited.

\begin{abstract}
The World Health Organization has declared the outbreak of COVID-19 as a global pandemic. The alarming levels of spread and severity of the viral disease has resulted in significant morbidity and mortality. Women often face the highest risk of suffering devastating losses from the pandemic. Historically, women's health has always been inadequately represented in responses to global outbreaks. Resources are often funnelled away from women's health services towards targets perceived to be more important. Pregnant women with suspected, probable or confirmed COVID-19, should have access to obstetric and foetal medicine, neonatal care as well as mental health and psychosocial support, at facilities ready to tackle maternal and neonatal complications. In this article, we attempt to look at the challenges faced by gynaecologists because of this pandemic, and provide an overview on the current protocols in antenatal care, foetal care, childbirth, and oncological care.
\end{abstract}

Keywords: Antenatal care, COVID-19, Fetal medicine, Gynaecologic oncology, Pandemic, Women's health

\section{INTRODUCTION}

On March 11, 2020, the World Health Organization (WHO) declared Coronavirus disease (COVID-19) a global pandemic. The WHO called for governments of countries to take urgent and aggressive steps to stop the spread of the severe acute respiratory syndrome Coronavirus 2 (SARS-CoV-2), which was first identified in December 2019 in Wuhan, China.

Owing to the alarming rate of spread and severity of the disease; as of August 25, 2020, globally over 23 million people were infected and over 810,000 people have lost their lives. This pandemic has left communities around the globe struggling with overwhelmed healthcare systems and large populations in quarantine. Global health authorities while battling the pandemic are also trying to prevent severe consequences for women's health.

Women are among the most vulnerable groups that face the highest risk of suffering devastating losses from the pandemic. Historically, women's health has not received much importance in the health response to global outbreaks. In order to throw light upon the myriad problems faced by a gynecologist, we have to further categorize these issues. Gynecologist often face predicaments in terms of antenatal care, fetal care and childbirth and finally in oncological care. 
Since antenatal care is an essential service, maternity care providers should continue to provide client-centered, respectful skilled care to ensure continued support for women with multiple complex needs. Maternity care has been repeatedly shown to be essential, and studies have shown that if women do not have access to antenatal services they are at increased risk of maternal death, stillbirth, and other adverse perinatal outcomes. ${ }^{1,2}$ Women living with adversity including poverty, homelessness, substance misuse, being an asylum seeker, experiencing domestic abuse and mental health problems will continue to require timely expert support. ${ }^{3}$

Pregnant women with suspected, probable or confirmed COVID-19, should have access to woman-centred care, including midwifery, obstetric, foetal medicine and neonatal care, as well as mental health and psychosocial support, at a centre ready to care for maternal and neonatal complications.

\section{IMPACT OF COVID-19 ON ANTENATAL CARE}

According to available data, pregnancy is not known to increase the risk of contracting COVID-19. ${ }^{4}$ A British study has shown that the proportion of pregnant women hospitalised was similar to the proportion in the general population, and pregnancy was not associated with increased mortality, unlike in influenza. ${ }^{5}$ Most women experienced only mild or moderate symptoms like cough, fever, shortness of breath, headache, and anosmia. ${ }^{3}$ If the pregnant lady has co-morbid conditions such as diabetes, hypertension, obesity, or she is of advanced age, she is more likely to have a severe form of respiratory disease. ${ }^{6}$

Pregnancy is a known to cause a hypercoagulable state, and there is emerging evidence suggesting that individuals with COVID-19 also develop hypercoagulable state. This combination could potentially increase the risk of maternal venous-thromboembolism. ${ }^{7}$ Reduced mobility resulting from self-isolation at home or hospital admission is likely to increase the risk further. ${ }^{3}$

The guidelines issued by the Indian Council of Medical Research (ICMR) and Royal College of Obstetricians and Gynaecologists (RCOG) advise women to attend routine antenatal care unless they meet self-isolation criteria like continuous cough or fever. WHO has recommended that antenatal or postnatal counselling and follow up should be conducted via alternative platforms such as telemedicine. ${ }^{8}$

\section{IMPACT OF COVID-19 ON FOETAL MEDICINE}

Due to the limited number of COVID-19 infected women who have gone into labour, there is insufficient evidence of it causing any foetal abnormalities or alteration of other foetal parameters of growth, amniotic fluid or Doppler indices. Currently, there is no evidence COVID19 infection is associated with a higher risk of abortion. However, as with other systemic diseases which can compromise maternal health, there is a possibility of preterm labour. ${ }^{6}$

According to RCOG, there is no increased risk of miscarriage or early pregnancy loss in relation to COVID-19. Currently, there is no evidence that the virus is teratogenic either. ${ }^{5}$ However, recent evidence has suggested that it is probable that the virus can be vertically transmitted, although the proportion of pregnancies affected and the risk to the foetus is yet to be determined. ${ }^{9,10}$

With the current uncertainty regarding the potential effects of COVID-19 infection on foetus, the methods of providing a regular antenatal care, foetal ultrasound imaging and counselling has changed. ${ }^{11}$ Most foetal therapies and screening are time bound and cannot be delayed. Only after routine triaging and risk assessment of COVID-19 transmission, should a reference to foetal medicine unit be considered.

However, high risk pregnancies (for example, previous child with known single gene disorder, non-invasive prenatal testing high risk for T21/T18/T13, COVID-19 positive, pre-GDM with poor perinatal glycaemic control, suspected major foetal anomaly, reduced foetal movements) that require foetal therapy need to be seen immediately. ${ }^{12,13}$

\section{Change in ultrasound visits}

Instead of a routine viability scan, a first trimester ultrasound can be done between 11-14 weeks. This scan can be used to date the pregnancy, confirm viability, exclude major structural abnormalities, assess nuchal translucency, and simultaneously blood can be drawn for routine antenatal, biochemical, or non-invasive prenatal screening. ${ }^{14}$

If there are no obstetric high-risk factors, growth scans can be done at 34-36 weeks instead of the routine timing of 32 weeks. In case of lethal anomalies, the option of termination of pregnancy should be offered after discussion with the obstetric team.

Reduced appointments and triaging can result in delay of target scan. In low risk mothers, adequate reassurance should be made that the chance of missing a major problem is low. This can be done through teleconsultation, as the risk of travelling during a pandemic could potentially be higher than detecting a problem on an ultrasound.

\section{Fetal therapy and procedures}

A study has shown that SARS-CoV-2 was not detected in amniotic fluid or umbilical cord blood of women with COVID-19. ${ }^{15}$ Minimally invasive procedures like amniocentesis may be preferable to chorionic villus sampling. ${ }^{14}$ A COVID-19 screening for the mother needs 
to be done before taking up the procedure. If found to be positive, then the procedure should be delayed.

A symptomatic mother may need further clinical evaluation using imaging modality like X-ray or chest CT. The most common adverse effects due to high-dose radiation exposure that occurs during chest imaging, especially CT scan are foetal growth restriction, microcephaly and intellectual disability. ${ }^{16-20}$

Pregnant women with suspected or confirmed COVID-19 infection who are either asymptomatic or recovering from mild illness, should be monitored with 2-4 weekly ultrasound assessment of foetal growth and amniotic fluid volume, with umbilical artery Doppler if necessary. ${ }^{21}$

Foetal medicine units must periodically review number of cases detected locally and adjust their guidelines and services accordingly. A pre-procedure counselling regarding the increase in scanning time due to PPE usage, difficulty in operating the probes with the PPE, increase in inter-scan intervals due to fumigation, probe cleaning procedures, reduced number of appointments and delay in scheduling them, missing the first trimester screening and option for non-invasive method of screening should be explained.

\section{MANAGEMENT IN A PREGNANT WOMAN}

If a pregnant woman has been exposed to SARS-CoV-2, clinical examination and RT-PCR (SARS-CoV-2) on deep nasopharyngeal and pharyngeal samples need to be done. ${ }^{22}$ If the mother is asymptomatic, we have to advise home monitoring of temperature and respiratory symptoms. If SARS-CoV-2 negative, we can cease monitoring. However, if she is SARS-CoV-2 positive, we need to advise isolation at home for 14 days.

If the expectant mother has a temperature of $>38^{\circ} \mathrm{C}$ and has respiratory symptoms, she is to be monitored at hospital. She should be in an isolation room with negative pressure (IRNP). If SARS-CoV-2 negative, we can advise home isolation for 14 days with self-monitoring. If symptoms persist, retest her. However, if she is SARSCoV-2 positive, we need to hospitalise her for maternal and foetal surveillance. Even her delivery should preferably take place at a tertiary care centre. ${ }^{22}$

At the time of admission, staff wearing appropriate personal protective equipment (PPE) should be present at the entrance of the maternity unit and provide these women with a surgical face mask. Until the woman is isolated in a suitable room, this mask should not be removed. Isolation rooms should have a defined area for staff to don and doff PPE with bathroom facilities. Only essential staff should enter the room and visitors should be strictly kept to a minimum. All clinical areas that are used must be cleaned after use.
Once admitted, assessment of the severity of COVID-19 symptoms should be done. The assessment should be done by a multidisciplinary team which includes an infectious disease or medical specialist. ${ }^{22}$

In pregnant women suspected to be COVID-19 positive, there should be no delay in starting therapy. ${ }^{3}$ The use of tocolytics is contraindicated in preterm labour and use of beta-mimetic agents should be avoided if there is pulmonary involvement. Use of glucocorticoids have been associated with an increased risk for mortality in patients with influenza. ${ }^{6}$

The timing of delivery should not be altered due to the COVID-19 infection. ${ }^{6}$ The National Institute for Health and Care Excellence (NICE) recommends avoiding induction of labour where it is not medically indicated and suggested on improving provisions for outpatient induction of labour. ${ }^{23}$ According to WHO, interventions to accelerate labour and childbirth (like augmentation, episiotomy, operative vaginal birth) should only be undertaken if medically justified and based on the clinical condition of mother and foetus.

Intrapartum services should be provided in a safe way, while keeping staffing requirements to a minimum and retaining the ability to provide emergency obstetric, anaesthetic and neonatal care when needed. ${ }^{3}$ Adequate counselling of mother, minimising number of people in the labour room, providing proper PPE for staff and adherence to strict hygienic practices are also of utmost importance.

According to WHO, mode of birth should be based on obstetric indications and the woman's preference, and caesarean section should only be undertaken when medically justified and based on maternal and foetal condition. Federation of obstetric and gynaecological societies of India (FOGSI) guidelines have mentioned that there is no evidence to favour one mode of birth over another.

A study has observed negative viral test results in vaginal secretion specimens, suggesting that a vaginal delivery may be a safe delivery option. ${ }^{24}$ In the same study, neonatal throat swabs were also negative. Taken together, these findings suggest that the risk of vertical transmission from pregnant women to newborn delivered by caesarean section is low.

Even though presence of COVID-19 infection is not an indication for caesarean section, universally COVID-19 positive women have been undergoing caesarean section when they present in labour. There is no proven scientific rationale for offering caesarean section due to COVID-19 infection. ${ }^{25}$ Unless the woman's respiratory condition demands urgent delivery, mode of birth should not be influenced by the presence of COVID-19. ${ }^{6}$ In case a woman has respiratory involvement due to COVID-19 infection, the second stage of delivery should be 
shortened to reduce maternal efforts and prevent maternal exhaustion.

There has been contention regarding the timing of clamping the cord. The American College of Obstetricians and Gynecologists (ACOG) recommends immediate cord clamping, whereas RCOG and WHO recommends delayed cord clamping. ${ }^{26}$ Delayed umbilical cord clamping is recommended to improve maternal and infant health. There is insufficient evidence to show that delayed cord clamping increases the possibility of viral transmission from mother to newborn. ${ }^{3}$ The proven benefits of a 1 to 3-minute delay in clamping the cord outweighs any unproven harms.

Starting prophylactic antibiotics is also strongly recommended. As the team needs to don PPE, there may be some delay in starting the emergency caesarean and this should be explained to the patient and family. Postop care should include principles of isolation room and barrier nursing with universal standard precautions.

\section{IMPACT OF COVID-19 ON THE NEWBORN}

Newborns of confirmed or suspected COVID-19 mothers present a new set of challenges for clinicians, especially when it comes to preventing perinatal transmission of the virus. The American academy of paediatrics committee on foetus and newborn highlighted that there is no data available to determine the possibility of transplacental transmission of the virus, any maternal body fluids carrying the Coronavirus, and any maternal, intrapartum and neonatal factors causing perinatal transmission. ${ }^{27}$

The available data suggests low rates of peripartum transmission and does not provide answers on the possible in-utero transmission of SARS-CoV-2 from expectant mothers with COVID-19 to their newborns. This gives us no means to determine the possible risks. Neonates born to pregnant women with confirmed COVID-19 or with test-pending at the time of delivery should be considered as persons under investigation. ${ }^{28}$

\section{At the time of delivery}

Delivery of a baby born to a COVID-19 mother increases the chances of maternal virus aerosols. In case of neonatal resuscitation and stabilisation, clinicians should use the PPE to prevent exposure to maternal virus aerosols and to neonatal aerosols generated. Infants born to COVID-19 women should be tested for SARS-CoV-2 at 24 hours. If the baby is still in the postnatal ward, then the test should be done at 48 hours after birth. ${ }^{28}$

\section{Maternal and newborn separation}

The infected mother and her newborn should be temporarily separated to lower the risk of postnatal infection caused due to the mother's respiratory secretions. This precautionary separation should be informed to the mother and discussed with her family before delivery.

\section{Neonatal care after maternal separation}

Infants that are born at or near term, and seem to be doing well at birth may be kept in a separate area from newborns unaffected by maternal COVID-19. Newborns are to be bathed within a reasonable period after birth so as to eliminate any possible viral presence on skin. Newborns that need intensive neonatal care should be kept in a single patient room with the potential for negative room pressure (or other air filtration systems).

Even after hospital discharge, a confirmed COVID-19 mother should follow some standard precautionary measures and avoid proximity to the infant. Relative who will take care of baby at home may be identified for baby care as early as possible to avoid delay in baby discharge. $^{29}$ If a newborn requires neonatal care, the physician should advise strict physical separation between the infected mother and the infant.

\section{Breastfeeding}

Studies conducted so far do not provide any evidence of breast milk containing SARS-CoV-2. ${ }^{30}$ Infected mothers can express breast milk but only after exercising appropriate breast and hand hygiene. She must use properly cleaned breast pump and accessories.

Mother should wear a well-fitting mask and a gown or towel to cover her clothes before feeding. Mother should wear disposable gloves if possible. A designated caregiver for the infant should feed the baby milk collected in a clean bottle. The caregiver should ensure the pump and accessories are cleaned after every use using disinfectant wipes and hot soapy water.

\section{IMPACT OF COVID-19 ON GYNAECOLOGIC ONCOLOGY}

Is it safe for my sister to come to the hospital? What about her treatment? asked the relative of a newly diagnosed case of endometrial adenocarcinoma. Such questions have become all too common during this pandemic. This pandemic has caused a wave of anxiety and fear among patients throughout the world, mostly among those with chronic illness and more specifically, those with cancer.

The WHO-China Joint commission report was among the first to highlight the details of COVID-19 infection and its fatality. It was noted that older individuals (above 60 years), and those with co-morbidities had a higher risk of mortality. ${ }^{31}$ Cancer is known to weaken the immune system, both the innate and acquired immunity. The immunosuppression is even more marked in those undergoing chemotherapy, radiation or surgery for treatment, making them more susceptible to various 
infections. $^{32-35}$ Thus, contracting COVID-19 infection will further worsen the prognosis. This was substantiated by a multicentric study revealing adverse outcomes in individuals with both cancer and COVID-19 infection, with a death rate of $11.43 \% .^{36}$

Another report from China has retrospectively analysed the clinical features, treatment and outcome of three women with gynaecological cancers who tested COVID19 positive. Two of them were recovered, while one succumbed to it, thus reinforcing that women with gynaecological malignancies are susceptible for COVID19 infection and can deteriorate rapidly. ${ }^{37}$

Another important concern to address is the risk of delay in diagnosis and treatment. A study analysing the effect of delay in treatment of cervical cancer patients, showed a 2.31 times increased risk of mortality when there was delay of more than or equal to 4 months than those who underwent timely treatment. ${ }^{38}$

As for endometrial cancer, a period of more 6 weeks delay from diagnosis to treatment was considered to have a negative impact on survival. ${ }^{39}$ Ovarian malignancy also showed similar detrimental outcome to overall survival with delay in treatment of more than 6 weeks, especially in those with advanced stage of the disease. ${ }^{40}$

Paradoxically, delaying chemotherapy or surgery worsens prognosis, but both modalities cause immunosuppression, thereby increasing the risk of COVID-19 infection. ${ }^{33,24,41}$ Aerosol generating procedures during surgery such as intubation for general anaesthesia further increases the risk of transmission of the virus. ${ }^{42,43}$

In patients with pre-invasive lesions or slow growing cancers, postponement of surgery may be considered. Laparoscopic surgeries should be discouraged during this outbreak. $^{44}$ Type and stage of cancer, other medical comorbidities and prevalence of COVID-19 cases in the area should be taken into consideration while prioritizing cases, planning treatment, and finalizing whether early treatment or delaying treatment would improve survival outcome. $^{45}$

To assist oncologists in this decision-making process, many centres have provisionally put up guidelines. British Gynaecological Cancer Society developed a framework for managing various type of gynaecological cancers. The Society of Gynecologic Oncology also put up guidelines for surgical prioritization, classifying indication for gynaecological surgeries into urgent, semiurgent and non-urgent. ${ }^{46,47}$

Urgent/emergent; urgent procedures refer to procedures that is acutely time-sensitive and performed when the patient is medically stable. Emergent procedures refer to those performed without delay to preserve life or limb. These are performed immediately. These include surgeries for viscus perforation, closed-loop bowel or colonic obstruction, incarcerated hernia with gynaecologic tumour, vaginal, uterine or pelvic haemorrhage, molar pregnancy, and pelvic mass with torsion or with urinary or intestinal obstruction.

Semi-urgent; these are performed in order to preserve the patient's life or prevent expected progression of disease or morbidity. These are performed in 1-4 weeks. These include cases of established cancer diagnosis when high suspicion exists, grade 1 endometrial cancer when hormonal therapy is contraindicated or not possible, all stages of high-grade uterine cancers, cervical and vulvar cancers, advanced ovarian cancer, abdominopelvic masses concerning for malignancy, symptomatic gynaecologic cancer in pregnancy requiring surgery, patients with recurrent disease without non-surgical options, and symptomatic patients with inoperable primary or recurrent cancer requiring palliative cancer procedures.

Non-urgent; procedure are deemed non-urgent if progression of disease or symptoms, or readmission within 3 months is unlikely, or if non-surgical treatments are available. These include benign-appearing ovarian cysts or masses, VAIN (vaginal intra-epithelial neoplasia)/VIN (vulvar intraepithelial neoplasia) 2-3, CIN (cervical intraepithelial neoplasia) 2-3, CAH/EIN (complex atypical hyperplasia/endometrial intraepithelial neoplasia), grade 1 endometrial cancer when hormonal therapy is not contraindicated, and completion surgery for early-stage.

Highest priority should be given for surgical treatment of aggressive cancers with an increased predilection for early metastases and symptom progression. This is especially important to prioritize those that can be managed in a minimally invasive/short stay manner.

Availability of ICU and ventilators during this period is another concern. Most of these resources have been diverted towards managing COVID-19 cases. Major surgeries or procedures on high-risk patient with other medical comorbidities which may requiring ICU care is best postponed. Patients with advanced cancers and those intended for palliative treatment, surgery best postponed for them and instead neoadjuvant and palliative chemotherapy respectively.

The current exponential rise of cases is causing shortage of resources, especially PPE for healthcare workers. Increase in strain on the manpower in the health care sector is another concern. Many health care workers have tested positive for COVID-19 leading shortage of staff and longer working shifts and rising tension at the workplace. Anxiety of patients has also led to rising incidents of violence against doctors.

Patients should be provided emergency contact numbers and given pamphlets providing information about the disease, symptoms and measures to protect 
themselves. $^{48,49}$ Considering the current crisis, the Medical Council of India has released guidelines for telemedicine consults. ${ }^{50}$

\section{IMPACT OF COVID-19 ON WOMEN'S HEALTH}

Historically in a pandemic, resources are often funnelled away from reproductive health services towards targets perceived to be more important. ${ }^{51}$ However even in these trying times, keeping the spotlight on women's health is vital, as safe pregnancies and childbirth depend on functioning and accessible health care systems.

Like China, India had announced a complete lockdown of all activities from 24 March 2020 to contain the spread of COVID-19. ${ }^{52}$ The poorly planned lockdown has caused unprecedented hardships for several vulnerable groups, including pregnant women and cancer patients.

Scientifically sound strategies need to be implemented in order to mitigate the potentially catastrophic fallout of this pandemic. We have seen the effect of the 2014-2016 Ebola outbreak in West African nations. In Sierra Leone, the Ebola outbreak caused maternal deaths to soar back to rates observed during the Sierra Leone civil war (19912002). ${ }^{53}$ The country is still struggling to recover from the effects of that outbreak. We need to ensure the same is not true for our country when the effects of COVID-19 become clear.

We need to prioritise continued access to family planning care, maintenance of supply chains, and targeted information campaigns about the transmission of COVID-19.

\section{CONCLUSION}

While it is right to focus on the management of COVID19 to mitigate the long-term consequences, we should also continue to focus on providing adequate safe and appropriate obstetric and gynaecological care. The lockdown has forced the entire nation to remain indoors for months; this could see an increase in the number of pregnancies in the coming months which will test the limits of our health system. Due to the lack of access to health care during the lockdown many women would not have gone to their doctors for timely screenings. This could result in a higher number of women diagnosed with more advanced cancers. Lockdowns, travel restrictions, supply chain disruptions, the huge shift of health resources to combat COVID-19 and fear of infection continue to prevent many women and girls from care. The national and state bodies should anticipate this in the light of the current pandemic and issue appropriate guidelines. To allay the anxiety and concerns of patients and relatives, they should be counselled and educated about the disease and about measures to protect themselves and when to seek treatment.

\section{ACKNOWLEDGMENTS}

The authors would like to thank Dr. Abi Manesh for his critical inputs and valuable insights.

Funding: No funding sources

Conflict of interest: None declared

Ethical approval: Not required

\section{REFERENCES}

1. Dowswell T, Carroli G, Duley L, Gates S, Gülmezoglu AM, Khan-Neelofur D, Piaggio G. Alternative versus standard packages of antenatal care for low-risk pregnancy. Cochrane Database Syst Rev. 2015;2015(7):CD000934.

2. Knight M, Bunch K, Tuffnell D. Saving lives, improving mothers' care. Available at: https://www.npeu.ox.ac.uk/mbrrace-uk/presentations/saving-lives-improving-mothers-care. Accessed on 20 June 2020.

3. Coronavirus (COVID-19) infection in pregnancy. Available at: https://www.rcog.org.uk/globalassets/ documents/guidelines/2020-05-13-coronaviruscovid-19-infection-in-pregnancy.pdf. Accessed on 20 June 2020.

4. Raleigh VS, Hussey D, Seccombe I, Hallt K. Ethnic and social inequalities in women's experience of maternity care in England: results of a national survey. J R Soc Med. 2010;103(5):188-98.

5. Docherty $\mathrm{AB}$, Harrison EM, Green CA, Hardwick HE, Pius R, Norman L, et al. Features of 16,749 hospitalised UK patients with COVID-19 using the ISARIC WHO clinical characterisation protocol. medRxiv. 2020;2020:1-20.

6. GCPR on Pregnancy with COVID-19 infection. Available at: https://www.fogsi.org/wpcontent/ uploads/covid19/fogsi_gcpr_on_pregnancy_with_C OVID_19_version_1.pdf. Accessed on 20 June 2020.

7. Practical guidance for the prevention of thrombosis and management of coagulopathy and disseminated intravascular coagulation of patients infected with COVID-19. Available at: https://static1.squarespace. com/static/5e6613a1dc75b87df82b78e1/t/5eecb111f dd1e0249e31904a/1592570129615/VTE-Patientswith-COVID19.pdf. Accessed on 20 June 2020.

8. Pregnancy, childbirth and COVID-19. Available from: https://www.who.int/emergencies/diseases/ novel-coronavirus-2019/question-and-answershub/q-a-detail/q-a-on-covid-19-pregnancy-andchildbirth. Accessed on 20 June 2020.

9. Dong L, Tian J, He S, Wang J, Zhu C, Liu C, et al. Possible vertical transmission of SARS-CoV-2 from an infected mother to her newborn. JAMA. 2020; 323(18):1846-8.

10. Zeng H, Xu C, Fan J, Tang Y, Deng Q, Zhang W, et al. Antibodies in infants born to mothers with covid19 pneumonia. JAMA. 2020;323(18):1848-9.

11. Poon LC, Yang H, Lee JCS, Copel JA, Leung TY, Zhang Y, et al. ISUOG Interim guidance on 2019 
novel coronavirus infection during pregnancy and puerperium: information for healthcare professionals. Ultrasound Obstet Gynecol. 2020;55 (5):700-8.

12. Guidance for fetal medicine units (FMUs) in the evolving coronavirus (COVID-19) pandemic. Available at: https://www.rcog.org.uk/globalassets/ documents/guidelines/2020-07-10-guidance-forfetal-medicine-units.pdf. Accessed on 20 June 2020.

13. Safe practice in obstetric ultrasound and fetal medicine: COVID guidance. Available at: https://www.isuog.org/uploads/assets/dfd2171ac5ec-4fbc-94f33532c3eda67c/ISUOG-InterimGuidance-on-COVID-19-Update.pdf. Accessed on 20 June 2020.

14. Deprest J, Choolani M, Chervenak F, Farmer D, Lagrou K, Lopriore E, et al. Fetal Diagnosis and therapy during the COVID-19 pandemic: guidance on behalf of the International fetal medicine and surgery. Fetal Diagn Ther. 2020;1-10.

15. Chen H, Guo J, Wang C, Luo F, Yu X, Zhang W, et al. Clinical characteristics and intrauterine vertical transmission potential of COVID-19 infection in nine pregnant women: a retrospective review of medical records. Lancet. 2020;395(10226):809-15.

16. Li Y, Xia L. Coronavirus disease 2019 (COVID-19): role of chest ct in diagnosis and management. AJR Am J Roentgenol. 2020;214(6):1280-6.

17. Ai T, Yang Z, Hou H, Zhann C, Chen C, Lv W, et al. Correlation of chest ct and rt-pcr testing for coronavirus disease 2019 (COVID-19) in China: a report of 1014 cases. Radiology. 2020;296(2):E3240.

18. Patel SJ, Reede DL, Katz DS, Subramaniam R, Amorosa JK. Imaging the pregnant patient for nonobstetric conditions: algorithms and radiation dose considerations. Radiographics. 2007;27(6): 1705-22.

19. Drugs and lactation database (LactMed). Available at: https://www.ncbi.nlm.nih.gov/books/NBK50 1398. Accessed on 20 June 2020.

20. Miller RW. Discussion: severe mental retardation and cancer among atomic bomb survivors exposed in utero. Teratology. 1999;59(4):234-5.

21. Favre G, Pomar L, Qi X, Nielsen-Saines K, Musso $\mathrm{D}$, Baud D. Guidelines for pregnant women with suspected SARS-CoV-2 infection. Lancet Infect Dis. 2020;20(6):652-3.

22. Guidance for management of pregnant women in COVID-19 pandemic. Available at: https://www. icmr.gov.in/pdf/covid/techdoc/Guidance_for_Manag ement_of_Pregnant Women_in_COVID 19_Pandemic_12042020.pdf. Accessed on 20 June 2020.

23. Inducing labour. Available at: https://www.nice. org.uk/guidance/cg70/resources/inducing-labour-pdf975621704389. Accessed on 20 June 2020.

24. Wu Y, Liu C, Dong L, Zhang C, Chen Y, Liu J, et al. Viral Shedding of COVID-19 in pregnant women.
Available at: https://ssrn.com/abstract $=3562059$. Accessed on 20 June 2020.

25. Liu D, Li L, Wu X, Zheng D, Wang J, Yang l, et al. Pregnancy and perinatal outcomes of women with coronavirus disease (covid-19) pneumonia: a preliminary analysis. AJR Am J Roentgenol. 2020; 215(1):127-32.

26. Novel Coronavirus 2019 (COVID-19).Available at: https://www.acog.org/clinical/clinicalguidance/practice-advisory/articles/2020/03/novelcoronavirus-2019. Accessed on 20 June 2020.

27. Puopolo KM, Hudak ML, Kimberlin DW, Cummings JJ. Management of Infants Born to Mothers with COVID-19. Available at: https://www.the-hospitalist.org/hospitalist/article/ 221157/pediatrics/management-infants-bornmothers-covid-19. Accessed on 20 June 2020.

28. Amatya S, Corr TE, Gandhi CK. Management of newborns exposed to mothers with confirmed or suspected COVID-19. J Perinatol. 2020;40:987-96.

29. Evaluation and management considerations for neonates at risk for coronavirus disease 2019 (COVID-19). Available from: https://www.cdc.gov/ coronavirus/2019-ncov/hcp/caring-fornewborns.html. Accessed on 20 June 2020.

30. Carvalho WB, Gibelli MABC, Krebs VLJ, Calil VMLT, Johnston C. Expert recommendations for the care of newborns of mothers with COVID19. Clinics (Sao Paulo). 2020;75:e1932.

31. Report of the WHO-China joint mission on coronavirus disease 2019 (COVID-19). Available at: https://www.who.int/publications/i/item/report-ofthe-who-china-joint-mission-on-coronavirus-disease2019-(covid-19). Accessed on 20 June 2020.

32. Kamboj M, Sepkowitz KA. Nosocomial infections in patients with cancer. Lancet Oncol. 2009;10(6):58997.

33. Li JY, Duan XF, Wang LP, Xu YJ, Huang L, Zhang $\mathrm{TF}$, et al. Selective depletion of regulatory $\mathrm{T}$ cell subsets by docetaxel treatment in patients with nonsmall cell lung cancer. J Immunol Res. 2014; 2014:286170.

34. Longbottom ER, Torrance HD, Owen HC, Hinds CJ, Pearce RM, Fragkou PC, et al. Features of postoperative immune suppression are reversible with interferon gamma and independent of interleukin-6 pathways. Ann Surg. 2016;264(2):3707.

35. Sica A, Massarotti M. Myeloid suppressor cells in cancer and autoimmunity. J Autoimmun. 2017;85: 117-25.

36. Dai M, Liu D, Liu M, Zhou F, Li G, Chen Z, et al. Patients with cancer appear more vulnerable to SARS-CoV-2: a multicenter study during the COVID-19 Outbreak. Cancer Discov. 2020;10(6): 783-91.

37. Zhang J, Peng P, Li X, Zha YF, Zhang GN, Zhang $Y$, et al. Management strategies for patients with gynecological malignancies during the outbreak of 
COVID-19. Zhonghua Fu Chan Ke Za Zhi. 2020;55 (4):221-6.

38. Shen SC, Hung YC, Kung PT, Yang WH, Wang YH, Tsai WC. Factors involved in the delay of treatment initiation for cervical cancer patients: A nationwide population-based study. Medicine (Baltimore). 2016; 95(33):e4568.

39. Strohl AE, Feinglass JM, Shahabi S, Simon MA. Surgical wait time: A new health indicator in women with endometrial cancer. Gynecologic Oncology. 2016;141(3):511-5.

40. Liu Y, Zhang T, Wu Q, Jiao Y, Gong T, Ma X, et al. Relationship between initiation time of adjuvant chemotherapy and survival in ovarian cancer patients: a dose-response meta-analysis of cohort studies. Sci Rep. 2017;7(1):9461.

41. Eggermont AM, Steller EP, Sugarbaker PH. Laparotomy enhances intraperitoneal tumor growth and abrogates the antitumor effects of interleukin-2 and lymphokine-activated killer cells. Surgery. 1987; 102(1):71-8.

42. Tran K, Cimon K, Severn M, Pessoa-Silva CL, Conly J. Aerosol generating procedures and risk of transmission of acute respiratory infections to healthcare workers: a systematic review. PLoS One. 2012;7(4):e35797.

43. Odor PM, Neun M, Bampoe S, Clark S, Heaton D, Patel A, et al. Anaesthesia and COVID-19: infection control. Br J Anaesth. 2020;125(1):16-24.

44. Ramirez PT, Chiva L, Eriksson AGZ, Frumovitz M, Fagotti A, Martin AG, et al. COVID-19 Global pandemic: options for management of gynecologic cancers. Int J Gynecol Cancer. 2020;30(5):561-3.

45. Burki TK. Cancer care in the time of COVID19. Lancet Oncol. 2020;21(5):628.

46. BGCS framework for care of patients with gynaecological cancer during the COVID-19 pandemic. Available at: https://www.bgcs.org.uk/
wp-content/uploads/2020/03/BGCS-covid-guidancev1.-22.03.2020.pdf. Accessed on 20 June 2020.

47. Fader AN, Huh WK, Kesterson J, Pothuri B, Wethington S, Wright JD, et al. When to operate, hesitate and reintegrate: society of gynecologic oncology surgical considerations during the COVID19 pandemic. Gynecol Oncol. 2020:158(2):236-43.

48. Coronavirus disease 2019 (COVID-19) - prevention \& treatment. Available at: https://www.cdc.gov/ coronavirus/2019-ncov/prevent-gettingsick/prevention.html. Accessed on 20 June 2020

49. Ueda M, Martins R, Hendrie PC, Crews JR, Wong TL, Jagels B, et al. Managing cancer care during the COVID-19 pandemic: agility and collaboration toward a common goal. J Natl Compr Canc Netw. 2020;1-4.

50. Telemedicine practice guidelines: enabling registered medical practitioners to provide healthcare using telemedicine. Available at: https://www.mohfw. gov.in/pdf/Telemedicine.pdf. Accessed on 20 June 2020.

51. Wenham C, Smith J, Morgan R. COVID-19: the gendered impacts of the outbreak. The Lancet. 2020;395(10227):846-8.

52. Modi Orders 3-Week total lockdown for all 1.3 billion Indians. Available at: https://www.nytimes. com/2020/03/24/world/asia/india-coronaviruslockdown.html. Accessed on 20 June 2020.

53. Davies SE, Bennett B. A gendered human rights analysis of Ebola and Zika: locating gender in global health emergencies. Int Aff .2016;92:1041-60.

Cite this article as: George AM, Manoj D, Ramani S, Lalropuii, Murugan TP, James RI, et al. Obstetrics and gynaecology in the era of COVID-19. Int $\mathbf{J}$

Reprod Contracept Obstet Gynecol 2020;9:4777-84. 